3. Increase staff understanding of discharge processes

4. Produce patient information leaflet.

Method A senior staff nurse led the work stream and undertook a retrospective base line audit of patient discharges and a staff survey to explore their experience of discharging patients from the in-patient unit (IPU). Using these results a patient leaflet has been produced, clearly outlining hospice expectations, discharge details and funding streams.A parttime discharge educator, with extensive experience of continuing health care, has been employed to review discharge processes and provide staff education. Education has been delivered one-to- one in relation to patient discharges in real time and within a formal setting.

Results Audit results have shown that the uncertainty of end of life care, family concerns and timely access to nursing home placements and care packages are significant factors when planning discharge from the IPU. It was also identified that clinical pressures affected timely completion of discharge documentation.

Achievements at six months:

- Increased patient discharges

- Reduction in average length of stay to 14 days

- All qualified staff have received training and awareness training for nursing assistants planned

- Patient leaflet completed.

Conclusion Patient flow and staff confidence have increased significantly following commencement of the project and the discharge educator post has been extended for an additional six months.

\section{P-163 USING ACUPUNCTURE TO TREAT HOT FLASHES AND NIGHT SWEATING FOR PATIENTS WITH BREAST CANCER}

Visweswaran Ramasamy. John Taylor Hospice, Birmingham, UK

\subsection{6/bmjspcare-2017-hospice. 188}

Background Hot flashes and night sweating are a common disruptive clinical problem that affects nearly two-thirds of all breast cancer survivors. Adjuvant hormonal therapies are an essential part of the treatment regimen for early breast cancer, and are used to prevent recurrence. However, hot flashes and night sweating are the most frequently occurring side effects of these interventions.

Aim To assess the effectiveness of acupuncture in breast cancer related hot flashes and sweating.

Methods A 44 year -old woman with a diagnosis of breast cancer had been suffering from intractable hot flashes and night sweating for the past two years. She had tried all other measures but these had very little or no effects on her symptoms. The subject received acupuncture treatment once a week for eight weeks, and the needles were stimulated manually every $10 \mathrm{~min}$ during each sessions. In all the treatment sessions, acupuncture points were stimulated manually and the needles were left in situ for 30 mins. Subjective measurement of vasomotor symptoms used Visual Analogue Scale (VAS).

Results A noticeable reduction in hot flashes and night sweating were observed after six sessions of treatment, which had a huge impact on her sleep pattern and psychological wellbeing.
By the end of eight weeks of treatment the subject reported a significant reduction in both her vasomotor and associated symptoms such as palpitations, anxiety, irritability and headaches.

Conclusion It would appear that eight sessions of acupuncture treatment had been shown to be effective in reducing vasomotor symptoms, especially for breast cancer patients who are seeking nonpharmacological therapies.

\section{P-164 THE ROLE OF KOREAN MEDICINE IN PALLIATIVE MEDICINE FOR PATIENTS WITH TERMINAL CANCER}

Hae-chang Yoon, Jung-hyo Cho. Dae-Jeon University, Daejeon, Republic of Korea

10.1136/bmjspcare-2017-hospice. 189

Background Modern palliative care began in the hospice movement led by Dr. Cicely Saunders. In Korea, it was started earlier but built the foundation recently regarding Korean medicine (KM). In 2015, the strategy for development of KM was established. It included the guidelines of cancer-related fatigue and anorexia.

Objectives The aim was to figure out the current state of hospice-palliative care (HPC) and evaluate the effectiveness of $\mathrm{KM}$ in terms of HPC.

Methods Systemic reviews published until Feb 2017. Literatures in international journals were searched on PubMed, MEDLINE, ScienceDirect and CINAHL; and articles in Korean journal on OASIS operated by the Korean Institute of Oriental Medicine. Keywords were palliative care with acupuncture, moxibustion and herb medicine in English and Korean

Results The results in acupuncture, moxibustion and herb medicine were 19,3 and 15 respectively. First, acupuncture was effective to relieve nausea and vomiting, anxiety, cancerrelated fatigue and leukopenia. Second, moxibustion was helpful for treating nausea and vomiting, cancer-related fatigue and leukopenia. Third, herb medicine was beneficial to improve quality of life and make the scales of immune system better. But there were some conflictive outcomes about other symptoms. Nevertheless, our view was there was no or slight adverse effect and most patients treated with KM were satisfied. Recently the number of hospitals and medical staff for HPC has been increasing but not enough compared with the number of patients who need HPC. Additionally the number of patients using $\mathrm{KM}$ has grown and the classification of them varied but the National Health Insurance didn't cover it sufficiently.

Conclusion The result of this review suggested that $\mathrm{KM}$ was effective for some symptoms related to terminal cancer patients. Therefore it may be the appropriate treatment for palliative care. This is the reason why we should make effort for $\mathrm{KM}$ to be more developed for end-of-life patients.

\section{P-165 SEPSIS CARE BUNDLE FOR A HOSPICE IN-PATIENT UNIT}

Paula Powell, Daniel Monnery, Susan Schofield. Willowbrook Hospice, Merseyside UK

10.1136/bmispcare-2017-hospice. 190 